\title{
$\phi$ meson mass and decay width in strange hadronic matter
}

\author{
Rajesh Kumar $\odot^{*}$ and Arvind Kumar ${ }^{\dagger}$ \\ Department of Physics, Dr. B. R. Ambedkar National Institute of Technology Jalandhar, Jalandhar 144011, Punjab, India
}

(Received 13 May 2020; revised 11 September 2020; accepted 12 October 2020; published 27 October 2020)

\begin{abstract}
The in-medium mass and decay width of the $\phi$ meson in hot asymmetric strange hadronic matter is calculated using an effective Lagrangian approach for the $\phi K \bar{K}$ interaction. The in-medium contributions of the $K \bar{K}$ loop to the $\phi$ meson self-energy are computed by the medium modified kaon and antikaon mass, which is evaluated using the chiral SU(3) model. To deal with the ultraviolet divergence, we regularize the loop integral of self-energy with a dipole form factor, and present the results for the range of cutoff mass $\Lambda_{c}=1-4 \mathrm{GeV}$. In chiral model calculations, for strange matter, we find that the mass of kaons and antikaons decreases with the increase in baryonic density, whereas the finite temperature causes an increase in the mass. We observed that the in-medium $\phi$ meson mass decreases slowly with baryonic density whereas the decay width increases rapidly. The results in the present investigation support a result in the literature which indicates a small downward shift in mass and a large broadening in the decay width. In the asymmetric strange hadronic matter, the study of the $\phi$ mesons can be relevant for compressed strange baryonic matter. The experimental observables such as dilepton spectra can perhaps be extracted from experiments in the future Facility for Antiproton and Ion Research (FAIR) facility at GSI.
\end{abstract}

DOI: 10.1103/PhysRevC.102.045206

\section{INTRODUCTION}

The study of hadron properties in strange asymmetric matter at finite temperature is of considerable interest, and leads to understanding the quark gluon plasma (QGP) phase diagram in strong interaction physics [1-9]. Experimentally, heavy ion-collisions (HICs) play an important role in the study of hot and dense matter in the region of nonperturbative QCD. In HICs, when two asymmetric nuclei collide, the fireball containing QGP comes into existence. Within a very short interval, the fireball expands and converts into an ensemble of particles which consists of nucleons, hyperons, and mesons, collectively known as hadronic matter [10]. In a hadronic medium, the baryons and mesons are the degrees of freedom, therefore only nonperturbative physics can be applied here. Furthermore, due to the presence of strange particles in the medium, it is very important to include the strangeness fraction while studying the properties of mesons in hadronic matter. We see significant progress toward understanding the properties of strange dense matter at moderate temperature with the construction of future experiments such as CBM at the GSI Facility for Antiproton and Ion Research (FAIR),

\footnotetext{
*rajesh.sism@gmail.com

†iitd.arvind@gmail.com
}

Published by the American Physical Society under the terms of the Creative Commons Attribution 4.0 International license. Further distribution of this work must maintain attribution to the author(s) and the published article's title, journal citation, and DOI. Funded by $S C O A P^{3}$. the Nuclotron-based Ion Collider Facility (NICA) at Dubna, Russia, and J-PARC in Japan [10,11].

The in-medium study of light vector mesons $(\rho, \omega$, and $\phi$ ) includes interest in theoretical and experimental research [12-21] because of their role in exploring dilepton production in HICs. Dilepton production is considered a promising observable because of its weak interaction with baryons and mesons [21-27]. We are motivated to study the properties of the $\phi$ meson, particularly because of (i) the strong interaction with nucleons and $u / d$ quarks $[19,28-30]$ and (ii) the interaction of $\phi$ mesons with strange baryons and mesons. In the literature, using QCD van der Waals forces, the $\phi$ meson is used to test the multi-gluon exchange theory [31] and has implications for understanding dark matter [32-34]. There is also a possibility of $\phi$-mesic nuclei formation due to the negative mass shift in nuclear matter [19,35-38]. The strong interaction of $\phi$ mesons with $u / d$ quarks occurs due to the interplay with $K \bar{K}$ pairs, therefore, the in-medium properties of the kaon and antikaon play a crucial role. Kaplan and Nelson initiated the study of in-medium properties of kaons and antikaons [39]. They observed the negative mass shift of antikaons in the neutron star medium and suggested the possibility of antikaon condensation. The downward mass shift comes from the strong attractive interaction of the antikaons with nucleons. Due to this attractive interaction, the effective energy of the mesons becomes low by transforming in the attractive scalar field. The annihilation of $K \bar{K}$ into a dilepton mainly proceeds through the $\phi$ meson, which helps us to understand the properties of the $\phi$ meson in the dense hadronic medium [21-27]. Due to the relevance of $K$ and $\bar{K}$ isospin doublets in heavy-ion collisions, many theoretical $[8,40-50]$ and experimental [51-54] investigations 
have been made. The free space antikaon-nucleon scattering amplitudes are obtained from the covariant unitarized chiral coupled-channel approaches, which include the method of partial waves systematically [46,49,55-58]. The evaluation of the kaon self-energy from this mechanism explains the $K^{-}$meson interaction in hadronic matter. At nuclear saturation density, an attractive potential of about 40 to $60 \mathrm{MeV}$ is obtained from these calculations. Furthermore, using the chiral SU(3) model, the properties of kaons and antikaons are studied in isospin asymmetric nuclear matter at finite temperature [59], and in strange matter at zero temperature [8].

The in-medium theoretical observations of the $\phi$ meson mass and the decay width have been studied extensively in the literature. Several authors have speculated on the small downward mass shift and broadening in the decay width of the $\phi$ meson [19,21,28,60-63]. By considering the contributions of the kaon-antikaon loop to the self-energy, Ko et al. used the chiral perturbation theory to calculate the density-dependent kaon mass and found that at nuclear saturation density, $\rho_{0}$, the $\phi$ meson mass decreases very little (at most $2 \%$ ), and the width has the value $\approx 25 \mathrm{MeV}$ [21]. Also, the observation of substantial broadening of the decay width has been made [21]. In Ref. [60], at $\rho_{0}$, Klingl et al. reported the downward $\phi$ -meson mass shift $(<1 \%)$ and the broadened decay width of $45 \mathrm{MeV}$. Using the QCD sum rule approach with the linear density approximation, Hatsuda and Lee computed the in-medium $\phi$ mass, and predicted a small decrease at the nuclear saturation density $[61,62]$. The large broadenings of the $\phi$ decay width of about $30 \mathrm{MeV}$ in Ref. [28] and 22 $\mathrm{MeV}$ in [63] were anticipated. Recently, in Ref. [19], CobosMartinez et al. reported the downward mass shift of $25 \mathrm{MeV}$ and the large broadening width of $32.8 \mathrm{MeV}$ at the nuclear saturation density. Experimentally, the large broadening of the in-medium decay width has been investigated [64-67]. At the nuclear saturation density, the KEK-E325 Collaboration computed a decrement in mass $(3.4 \%)$ and an increase in the in-medium decay width $(\approx 14.5 \mathrm{MeV})$ of the $\phi$ meson [64]. In Ref. [65] SPring8 reported a large value of $\phi N$ cross section in the medium, which results in the decay width of $35 \mathrm{MeV}$ and is consistent with the experimental data [66,67]. Further experimental efforts are needed to understand the $\phi$ meson in the medium.

In the present article, we report the results of the inmedium $\phi$ meson mass and decay width in hot asymmetric strange hadronic matter by taking into account the medium induced kaon and antikaon masses. The in-medium $K$ and $\bar{K}$ properties are incorporated by a chiral effective Lagrangian using the chiral $\mathrm{SU}(3)$ model $[8,68]$. We calculate the inmedium masses of $K$ and $\bar{K}$ mesons at finite temperatures in strange hadronic matter and the decay width of $\phi$ meson. The chiral model is a nonperturbative hadron based model to describe the in-medium properties of hadronic matter [5-7,59,69-79]. It has also been applied to study the effect of a magnetic field on the in-medium properties of quarkonia [10,74] and open charm mesons [68,79]. To study the $K \bar{K}$ loop contributions in the $\phi$ meson decay, we use the effective Lagrangian of the $\phi K \bar{K}$ interactions and solve the loop integral by using regularization techniques $[19,80]$. In the current work, we include the contri- butions from the $K$ and $\bar{K}$ loop by utilizing the in-medium masses $m_{K^{+}}^{*}, m_{K^{0}}^{*}, m_{K^{-}}^{*}$, and $m_{\bar{K}^{0}}^{*}$. These in-medium masses are different from those in works such as Refs. [19,35], where the $\bar{K}$ contribution to the $K \bar{K}$ loop was suppressed by equalizing the mass via the relation $m_{K}^{*}=m_{\bar{K}}^{*}$.

The layout of the present paper is as follows: In Sec. II A, we concisely discuss the methodology to obtain the inmedium scalar and vector fields in hyperonic matter. In the Sec. II B, we calculate the induced mass of the kaon and antikaon via the interactions of the chiral model fields. The theoretical approach to calculate the in-medium mass and decay width of the $\phi$ meson is discussed in Sec. II C. In Sec. III, quantitative results of the present findings are discussed, and, to summarize, we conclude our work in Sec. IV.

\section{METHODOLOGY}

We use the chiral SU(3) model to study the impact of isospin asymmetry and strangeness fraction on the scalar and vector fields. Further, the $\phi$-meson mass and decay width are calculated from the self-consistent Lagrangian approach. In the forthcoming subsections, we briefly describe the formalism used to obtain the results.

\section{A. The hadronic chiral SU(3) model}

The chiral SU(3) model comprises fundamental QCD features such as the trace anomaly and nonlinear realization of the chiral symmetry [10,68,69,81-84]. In this model, the meson-baryon interactions are explained through the exchange of the scalar and vector fields. $\sigma$ is a nonstrange scalar-isoscalar field and corresponds to the scalar meson $\sigma_{0}(500)$. This meson is composed of light $u$ and $d$ quarks $(u \bar{d})$.

$\zeta$ is a strange scalar-isoscalar field identified with the scalar meson with strange quark content $s \bar{s}$ [85] and plays an important role in strange matter. Further, the scalar-isovector field $\delta$ is introduced in the relativistic mean-field models to study the properties of isospin asymmetric matter. This field is identified with the meson having quark content $\sim(\bar{u} u-\bar{d} d)$. The $\zeta$ and $\delta$ mesons are generally identified with mass around $980 \mathrm{MeV}$ [85-87]. The nucleons and hyperons in the medium interact through the exchange of the scalar fields $\sigma, \zeta$, and $\delta$ along with the vector fields $\omega, \rho$, and $\phi$. The scalar fields contribute to the medium range attractive interactions and the vector fields contribute to the short range repulsion or attraction depending upon the nucleon-meson interactions. For example, as we will see later the vectorial Weinberg-Tomozawa term gives a repulsive contribution to the kaon-nucleon interactions and an attractive one for the antikaon-nucleon case. Moreover, the scalar dilaton field, $\chi$ denotes the glueball field, which is a hypothetical gluon field containing gluon particles. It is introduced in the chiral models to mimic the trace anomaly property of QCD $[69,88]$. To simplify, the effects of fluctuations near phase transitions are neglected by using the mean-field approximation $[68,70]$. The model is successful in explaining nuclear matter, hypernuclei, finite nuclei, and neutron stars [10,68,69,81-84]. 
The hadronic chiral effective Lagrangian is given as

$$
\mathcal{L}_{\text {chiral }}=\mathcal{L}_{\text {kin }}+\sum_{M=S, V} \mathcal{L}_{B M}+\mathcal{L}_{\text {vec }}+\mathcal{L}_{0}+\mathcal{L}_{S B}
$$

where $\mathcal{L}_{\text {kin }}$ denotes the kinetic energy term, $\mathcal{L}_{B M}$ is the baryonmeson interaction term, $\mathcal{L}_{\text {vec }}$ produces the vector meson mass through the interactions with the scalar mesons and contains the quartic self-interaction terms, and $\mathcal{L}_{0}$ and $\mathcal{L}_{S B}$ define the spontaneous chiral symmetry breaking and the explicit chiral symmetry breaking, respectively. The symbols $S$ and $V$ represents the scalar and vector mesons, respectively. There exist $D$-type (symmetric) and $F$-type (antisymmetric) couplings corresponding to the baryon-vector meson interaction terms. Here we have used the antisymmetric coupling $[7,8]$. As per the vector meson dominance model, the $D$-type coupling should be less, which also follows the universality principle [89]. We choose the medium parameters [8] to dissociate nucleons from the strange field $\phi$, which leads to an ideal mixing between $\phi$ and $\omega$.

By using the Euler-Lagrange equations for the $\sigma$, $\zeta, \delta, \omega, \rho, \phi$, and $\chi$ mesonic fields of the chiral model, we obtained the following coupled equations of motion:

$$
\begin{aligned}
& k_{0} \chi^{2} \sigma-4 k_{1}\left(\sigma^{2}+\zeta^{2}+\delta^{2}\right) \sigma-2 k_{2}\left(\sigma^{3}+3 \sigma \delta^{2}\right)-2 k_{3} \chi \sigma \zeta \\
& -\frac{d}{3} \chi^{4}\left(\frac{2 \sigma}{\sigma^{2}-\delta^{2}}\right)+\left(\frac{\chi}{\chi_{0}}\right)^{2} m_{\pi}^{2} f_{\pi}=\sum g_{\sigma i} \rho_{i}^{s} \\
& k_{0} \chi^{2} \zeta-4 k_{1}\left(\sigma^{2}+\zeta^{2}+\delta^{2}\right) \zeta-4 k_{2} \zeta^{3}-k_{3} \chi\left(\sigma^{2}-\delta^{2}\right) \\
& \quad-\frac{d}{3} \frac{\chi^{4}}{\zeta}+\left(\frac{\chi}{\chi_{0}}\right)^{2}\left[\sqrt{2} m_{K}^{2} f_{K}-\frac{1}{\sqrt{2}} m_{\pi}^{2} f_{\pi}\right]=\sum g_{\zeta i} \rho_{i}^{s} \\
& k_{0} \chi^{2} \delta-4 k_{1}\left(\sigma^{2}+\zeta^{2}+\delta^{2}\right) \delta-2 k_{2}\left(\delta^{3}+3 \sigma^{2} \delta\right)+2 k_{3} \chi \delta \zeta \\
& +\frac{2}{3} d \chi^{4}\left(\frac{\delta}{\sigma^{2}-\delta^{2}}\right)=\sum g_{\delta i} \tau_{3} \rho_{i}^{s} \\
& \left(\frac{\chi}{\chi_{0}}\right)^{2} m_{\omega}^{2} \omega+g_{4}\left(4 \omega^{3}+12 \rho^{2} \omega\right)=\sum g_{\omega i} \rho_{i}^{v} \\
& \left(\frac{\chi}{\chi_{0}}\right)^{2} m_{\rho}^{2} \rho+g_{4}\left(4 \rho^{3}+12 \omega^{2} \rho\right)=\sum g_{\rho i} \tau_{3} \rho_{i}^{v} \\
& \left(\frac{\chi}{\chi_{0}}\right)^{2} m_{\phi}^{2} \phi+8 g_{4} \phi^{3}=\sum g_{\phi i} \rho_{i}^{v}
\end{aligned}
$$

and

$$
\begin{aligned}
& k_{0} \chi\left(\sigma^{2}+\zeta^{2}+\delta^{2}\right)-k_{3}\left(\sigma^{2}-\delta^{2}\right) \zeta+\chi^{3}\left[1+\ln \left(\frac{\chi^{4}}{\chi_{0}^{4}}\right)\right]+\left(4 k_{4}-d\right) \chi^{3} \\
& -\frac{4}{3} d \chi^{3} \ln \left(\left(\frac{\left(\sigma^{2}-\delta^{2}\right) \zeta}{\sigma_{0}^{2} \zeta_{0}}\right)\left(\frac{\chi}{\chi_{0}}\right)^{3}\right)+\frac{2 \chi}{\chi_{0}^{2}}\left[m_{\pi}^{2} f_{\pi} \sigma+\left(\sqrt{2} m_{K}^{2} f_{K}-\frac{1}{\sqrt{2}} m_{\pi}^{2} f_{\pi}\right) \zeta\right]-\frac{\chi}{\chi_{0}^{2}}\left(m_{\omega}^{2} \omega^{2}+m_{\rho}^{2} \rho^{2}\right)=0 .
\end{aligned}
$$

In the above equations, the model parameter $k_{i}(i=1$ to 4$)$ is fitted to regenerate the vacuum values of the scalar fields [73], and $m_{\pi}, m_{K}, f_{\pi}$, and $f_{K}$ denote the masses and decay constants of pion and kaon, respectively. The values of these parameters, along with other coupling constants, are fitted in the model to reproduce the vacuum masses of the baryon octet and are tabulated in Table I. In the equations of motion, $\rho_{i}^{v}$ and $\rho_{i}^{s}$ characterize the vector and scalar densities of the $i$ th baryon ( $\left.i=p, n, \Lambda, \Sigma^{ \pm}, \Sigma^{0}, \Xi^{-}, \Xi^{0}\right)[8,10]$ and are defined through the relations

$$
\rho_{i}^{v}=\gamma_{i} \int \frac{d^{3} k}{(2 \pi)^{3}}\left(\frac{1}{1+\exp \left[\beta\left(E_{i}^{*}(k)-\mu_{i}^{*}\right)\right]}-\frac{1}{1+\exp \left[\beta\left(E_{i}^{*}(k)+\mu_{i}^{*}\right)\right]}\right)
$$

and

$$
\rho_{i}^{s}=\gamma_{i} \int \frac{d^{3} k}{(2 \pi)^{3}} \frac{m_{i}^{*}}{E_{i}^{*}(k)}\left(\frac{1}{1+\exp \left[\beta\left(E_{i}^{*}(k)-\mu_{i}^{*}\right)\right]}+\frac{1}{1+\exp \left[\beta\left(E_{i}^{*}(k)+\mu_{i}^{*}\right)\right]}\right)
$$

respectively, where $\beta=\frac{1}{k T}$ and $\gamma_{i}$ is the degeneracy factor. Further, in this model the isospin asymmetry and strangeness are incorporated through the parameters $\eta=$ $-\frac{\Sigma_{i} \tau_{3} \rho_{i}^{v}}{2 \rho_{B}}$ and $f_{s}=\frac{\Sigma_{i}\left|s_{i}\right| \rho_{i}^{v}}{\rho_{B}}$, respectively. The $\tau_{3},\left|s_{i}\right|$, and $\rho_{B}$ symbolize the isospin quantum number (third component), number of strange quarks, and the total baryonic density, respectively.

\section{B. Kaon and antikaon interactions in the chiral model}

In this subsection, we evaluate the in-medium mass of the $K(\bar{K})$ mesons via the dispersion relation [90] in hot asymmetric strange hadronic matter $[7,78]$. The scalar and vector fields modify the scalar and vector densities of the baryons which further change the self-energy of the kaons and antikaons.

The interaction Lagrangian density for the kaons and antikaons is written as [8]

$$
\begin{aligned}
\mathcal{L}_{K B}= & -\frac{i}{4 f_{K}^{2}}\left[\left(2 \bar{p} \gamma^{\mu} p+\bar{n} \gamma^{\mu} n-\bar{\Sigma}^{-} \gamma^{\mu} \Sigma^{-}+\bar{\Sigma}^{+} \gamma^{\mu} \Sigma^{+}-2 \bar{\Xi}^{-} \gamma^{\mu} \Xi^{-}-\bar{\Xi}^{0} \gamma^{\mu} \Xi^{0}\right)\left(K^{-}\left(\partial_{\mu} K^{+}\right)-\left(\partial_{\mu} K^{-}\right) K^{+}\right)\right. \\
& \left.+\left(\bar{p} \gamma^{\mu} p+2 \bar{n} \gamma^{\mu} n+\overline{\Sigma^{-}} \gamma^{\mu} \Sigma^{-}-\overline{\Sigma^{+}} \gamma^{\mu} \Sigma^{+}-\overline{\Xi^{-}} \gamma^{\mu} \Xi^{-}-2 \overline{\Xi^{0}} \gamma^{\mu} \Xi^{0}\right)\left(\bar{K}^{0}\left(\partial_{\mu} K^{0}\right)-\left(\partial_{\mu} \bar{K}^{0}\right) K^{0}\right)\right]
\end{aligned}
$$




$$
\begin{aligned}
& +\frac{m_{K}^{2}}{2 f_{K}}\left[(\sigma+\sqrt{2} \zeta+\delta)\left(K^{+} K^{-}\right)+(\sigma+\sqrt{2} \zeta-\delta)\left(K^{0} \bar{K}^{0}\right)\right] \\
& -\frac{1}{f_{K}}\left[(\sigma+\sqrt{2} \zeta+\delta)\left(\partial_{\mu} K^{+}\right)\left(\partial^{\mu} K^{-}\right)+(\sigma+\sqrt{2} \zeta-\delta)\left(\partial_{\mu} K^{0}\right)\left(\partial^{\mu} \bar{K}^{0}\right)\right] \\
& +\frac{d_{1}}{2 f_{K}^{2}}\left(\bar{p} p+\bar{n} n+\bar{\Lambda}^{0} \Lambda^{0}+\overline{\Sigma^{+}} \Sigma^{+}+\overline{\Sigma^{0}} \Sigma^{0}+\overline{\Sigma^{-}} \Sigma^{-}+\overline{\Xi^{-}} \Xi^{-}+\bar{\Xi}^{0} \Xi^{0}\right)\left(\left(\partial_{\mu} K^{+}\right)\left(\partial^{\mu} K^{-}\right)+\left(\partial_{\mu} K^{0}\right)\left(\partial^{\mu} \bar{K}^{0}\right)\right) \\
& +\frac{d_{2}}{2 f_{K}^{2}}\left[\left(\bar{p} p+\frac{5}{6} \bar{\Lambda}^{0} \Lambda^{0}+\frac{1}{2} \overline{\Sigma^{0}} \Sigma^{0}+\bar{\Sigma}^{+} \Sigma^{+}+\overline{\Xi^{-}} \Xi^{-}+\overline{\Xi^{0}} \Xi^{0}\right)\left(\partial_{\mu} K^{+}\right)\left(\partial^{\mu} K^{-}\right)\right. \\
& \left.+\left(\bar{n} n+\frac{5}{6} \bar{\Lambda}^{0} \Lambda^{0}+\frac{1}{2} \bar{\Sigma}^{0} \Sigma^{0}+\bar{\Sigma}^{-} \Sigma^{-}+\overline{\Xi^{-}} \Xi^{-}+\bar{\Xi}^{0} \Xi^{0}\right)\left(\partial_{\mu} K^{0}\right)\left(\partial^{\mu} \bar{K}^{0}\right)\right]
\end{aligned}
$$

where the first term is defined as the vectorial interaction term (Weinberg-Tomozawa term), which is obtained from the kinetic part of the interaction Lagrangian. The second term is obtained from the explicit symmetry breaking and the third term is acquired from the kinetic terms of the pseudoscalar meson of the chiral effective Lagrangian $[59,78]$. The fourth and fifth terms are called range terms, which basically arise from the baryon meson interaction Lagrangian of the chiral model [77,78]. We have

$$
\mathcal{L}_{d_{1}}^{B M}=\frac{d_{1}}{2} \operatorname{Tr}\left(u_{\mu} u^{\mu}\right) \operatorname{Tr}(\bar{B} B)
$$

and

$$
\mathcal{L}_{d_{2}}^{B M}=d_{2} \operatorname{Tr}\left(\bar{B} u_{\mu} u^{\mu} B\right)
$$

Above, $B$ denotes the baryon octet. The dispersion relation for the kaon and antikaon is obtained by the Fourier transformation of the interaction Lagrangian, which is given by

$$
-\omega^{2}+\vec{k}^{2}+m_{K(\bar{K})}^{2}-\Pi^{*}(\omega,|\vec{k}|)=0 .
$$

Here $\Pi^{*}$ symbolizes the in-medium self-energy of kaon and antikaon. The self-energy of the kaon isospin doublet, $\left(K^{+}, K^{0}\right)$, is explicitly given as

$$
\begin{aligned}
\Pi_{K}^{*}(\omega,|\vec{k}|)= & -\frac{1}{4 f_{K}^{2}}\left[3\left(\rho_{p}^{v}+\rho_{n}^{v}\right) \pm\left(\rho_{p}^{v}-\rho_{n}^{v}\right) \pm 2\left(\rho_{\Sigma^{+}}^{v}-\rho_{\Sigma^{-}}^{v}\right)-\left[3\left(\rho_{\Xi^{-}}^{v}+\rho_{\Xi^{0}}^{v}\right) \pm\left(\rho_{\Xi^{-}}^{v}-\rho_{\Xi^{0}}^{v}\right)\right]\right] \omega+\frac{m_{K}^{2}}{2 f_{K}}\left(\sigma^{\prime}+\sqrt{2} \zeta^{\prime} \pm \delta^{\prime}\right) \\
& +\left[-\frac{1}{f_{K}}\left(\sigma^{\prime}+\sqrt{2} \zeta^{\prime} \pm \delta^{\prime}\right)+\frac{d_{1}}{2 f_{K}^{2}}\left(\rho_{s}^{p}+\rho_{s}^{n}+\rho_{\Lambda^{0}}^{s}+\rho_{\Sigma^{+}}^{s}+\rho_{\Sigma^{0}}^{s}+\rho_{\Sigma^{-}}^{s}+\rho_{\Xi^{-}}^{s}+\rho^{s} \Xi^{0}\right)\right. \\
& +\frac{d_{2}}{4 f_{K}^{2}}\left(\left(\rho_{p}^{s}+\rho_{n}^{s}\right) \pm\left(\rho_{p}^{s}-\rho_{n}^{s}\right)+\rho_{\Sigma^{0}}^{s}+\frac{5}{3} \rho_{\Lambda^{0}}^{s}+\left(\rho_{\Sigma^{+}}^{s}+\rho_{\Sigma^{-}}^{s}\right) \pm\left(\rho_{\Sigma^{+}}^{s}-\rho_{\Sigma^{-}}^{s}\right)\right. \\
& \left.\left.+2 \rho_{\Xi^{-}}^{s}+2 \rho_{\Xi^{0}}^{s}\right)\right]\left(\omega^{2}-\vec{k}^{2}\right)
\end{aligned}
$$

where the \pm signs indicate the self-energy for $K^{+}$and $K^{0}$ respectively. In the above expression the fluctuations $\sigma^{\prime}\left(=\sigma-\sigma_{0}\right)$, $\zeta^{\prime}\left(=\zeta-\zeta_{0}\right)$, and $\delta^{\prime}\left(=\delta-\delta_{0}\right)$ indicate the digression of the field expectation values from the vacuum expectation values. Also, $m_{K(\bar{K})}$ in Eq. (14) denotes the vacuum mass of the kaon (antikaon).

Similarly, the in-medium self-energy for the antikaon isospin doublet, $\left(K^{-}, \overline{K^{0}}\right)$, is evaluated as

$$
\begin{aligned}
\Pi_{\bar{K}}^{*}(\omega,|\vec{k}|)= & \frac{1}{4 f_{K}^{2}}\left[3\left(\rho_{p}^{v}+\rho_{n}^{v}\right) \pm\left(\rho_{p}^{v}-\rho_{n}^{v}\right) \pm 2\left(\rho_{\Sigma^{+}}^{v}-\rho_{\Sigma^{-}}^{v}\right)-\left[3\left(\rho_{\Xi^{-}}^{v}+\rho_{\Xi^{0}}^{v}\right) \pm\left(\rho_{\Xi^{-}}^{v}-\rho_{\Xi^{0}}^{v}\right)\right]\right]+\frac{m_{\bar{K}}^{2}}{2 f_{\bar{K}}}\left(\sigma^{\prime}+\sqrt{2} \zeta^{\prime} \pm \delta^{\prime}\right) \\
& +\left[-\frac{1}{f_{\bar{K}}}\left(\sigma^{\prime}+\sqrt{2} \zeta^{\prime} \pm \delta^{\prime}\right)+\frac{d_{1}}{2 f_{\bar{K}}^{2}}\left(\rho_{s}^{p}+\rho_{s}^{n}+\rho_{\Lambda^{0}}^{s}+\rho_{\Sigma^{+}}^{s}+\rho_{\Sigma^{0}}^{s}+\rho_{\Sigma^{-}}^{s}+\rho_{\Xi^{-}}^{s}+\rho_{\Xi^{0}}^{s}\right)\right. \\
& +\frac{d_{2}}{4 f_{K}^{2}}\left(\left(\rho_{p}^{s}+\rho_{n}^{s}\right) \pm\left(\rho_{p}^{s}-\rho_{n}^{s}\right)+\rho_{\Sigma^{0}}^{s}+\frac{5}{3} \rho_{\Lambda^{0}}^{s}+\left(\rho_{\Sigma^{+}}^{s}+\rho_{\Sigma^{-}}^{s}\right) \pm\left(\rho_{\Sigma^{+}}^{s}-\rho_{\Sigma^{-}}^{s}\right)\right. \\
& \left.\left.+2 \rho_{\Xi^{-}}^{s}+2 \rho_{\Xi^{0}}^{s}\right)\right]\left(\omega^{2}-\vec{k}^{2}\right)
\end{aligned}
$$

with the \pm signs for $K^{-}$and $\bar{K}^{0}$ respectively.

In strange hadronic matter, the in-medium mass of the $K(\bar{K})$ meson is evaluated by solving Eq. (14) under the condition $m_{K(\bar{K})}^{*}=\omega(|\vec{k}|=0)$. The parameters $d_{1}$ and $d_{2}$ in the expression of the self-energies are taken as $2.56 / m_{K}$ and $0.73 / m_{K}$ respectively [75], fitted with the help of experimental 


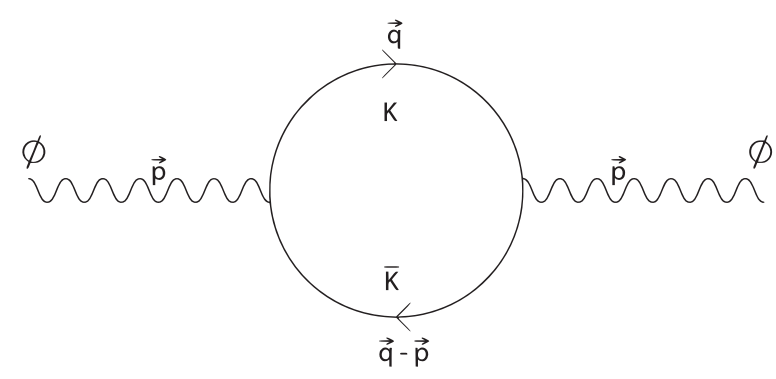

FIG. 1. The $\phi K \bar{K}$ interaction at the one loop level.

values of kaon-nucleon $(K N)$ scattering length [91]. In the present work, the vacuum values of $K^{+}\left(K^{-}\right)$and $K^{0}\left(\bar{K}^{0}\right)$ masses are taken as 494 and $495 \mathrm{MeV}$, respectively.

\section{The in-medium mass and decay width of the $\phi$ meson}

In this subsection, we compute the $\phi$ meson in-medium self-energy for the decay process $\phi \rightarrow K \bar{K}$ at the one-loop level (see Fig. 1). The interaction Lagrangian $\mathcal{L}_{\text {int }}[21,92]$ is given by

$$
\mathcal{L}_{\text {int }}=\mathcal{L}_{\phi K \bar{K}},
$$

where

$$
\mathcal{L}_{\phi K \bar{K}}=i g_{\phi} \phi^{\mu}\left[\bar{K}\left(\partial_{\mu} K\right)-\left(\partial_{\mu} \bar{K}\right) K\right] .
$$

In above equation, $g_{\phi}$ is the coupling constant and $K\left(K^{K^{+}}\right)$and $\bar{K}\left(K^{-}, \bar{K}^{0}\right)$ are the isospin doublets of kaons and antikaons. Note that in order to be consistent with calculations we used this notation for isospin doublets [19,59,93-95]. However, as per isospin doublet convention, the notation for $\bar{K}$ doublet is $\left(\bar{K}^{0},-K^{-}\right)$[63]. This convention follows the representation where particle with $-1 / 2$ isospin is considered at a lower position and results due to the rotation of isospin matrix.

In our present work, we have not considered the interactions of $\phi \phi K \bar{K}$ in the interaction Lagrangian due to the small contribution to the in-medium masses and decay width as compared to $\phi K \bar{K}$ interactions [19].

In the rest frame of the $\phi$ meson, the scalar part of the inmedium self-energy from the loop diagram is written as

$$
i \Pi_{\phi}^{*}(p)=-\frac{8}{3} g_{\phi}^{2} \int \frac{d^{4} q}{(2 \pi)^{4}} \vec{q}^{2} D_{K}(q) D_{\bar{K}}(q-p),
$$

where $\quad D_{K}(q)=\left(q^{2}-m_{K}^{*^{2}}+i \epsilon\right)^{-1} \quad$ and $\quad D_{\bar{K}}(q-p)=$ $\left((q-p)^{2}-m_{\bar{K}}^{*^{2}}+i \epsilon\right)^{-1}$ are the kaon and antikaon propagators, respectively; $p=\left(p^{0}=m_{\phi}^{*}, \overrightarrow{0}\right)$ is the $\phi$ meson four-momentum vector, with $m_{\phi}^{*}$ denoting the in-medium $\phi$ meson mass. The $m_{K}^{*}\left(=\frac{m_{K^{+}}^{*}+m_{K^{0}}^{*}}{2}\right)$ and $m_{\bar{K}}^{*}\left(=\frac{m_{K^{-}}^{*}+m_{K^{0}}^{*}}{2}\right)$ are the masses of kaon and antikaon, respectively. The values of $m_{K^{+}}^{*}, m_{K^{0}}^{*}, m_{K^{-}}^{*}$, and $m_{\bar{K}^{0}}^{*}$ will be solved using Eq. (14) and the in-medium mass of the $\phi$ is determined from the real part of $\Pi_{\phi}^{*}(p)$ by using the following relation [19]:

$$
m_{\phi}^{*^{2}}=\left(m_{\phi}^{0}\right)^{2}+\operatorname{Re} \Pi_{\phi}^{*}\left(m_{\phi}^{*^{2}}\right),
$$

where $m_{\phi}^{0}$ is the bare mass of the $\phi$ meson. The real part of the self-energy can be written as [19]

$$
\operatorname{Re} \Pi_{\phi}^{*}=-\frac{4}{3} g_{\phi}^{2} \mathcal{P} \int \frac{d^{3} q}{(2 \pi)^{3}} \vec{q}^{2} \frac{\left(E_{K}^{*}+E_{\bar{K}}^{*} v\right)}{E_{K}^{*} E_{\bar{K}}^{*}\left(\left(E_{K}^{*}+E_{\bar{K}}^{*}\right)^{2}-m_{\phi}^{*^{2}}\right)},
$$

where $\mathcal{P}$ denotes the principal value of the integral (19), $E_{K}^{*}=$ $\left(\vec{q}^{2}+m_{K}^{*^{2}}\right)^{1 / 2}$, and $E_{\bar{K}}^{*}=\left(\vec{q}^{2}+m_{\bar{K}}^{*^{2}}\right)^{1 / 2}$.

The integral in Eq. (21) is divergent. To avoid singularities, we regularize the integral by using the phenomenological form factor with the cutoff parameter $\Lambda_{c}$ [80]. The integral after regularization is given by

$$
\begin{aligned}
\operatorname{Re} \Pi_{\phi}^{*}= & -\frac{4}{3} g_{\phi}^{2} \mathcal{P} \int_{0}^{\Lambda_{c}} \frac{d^{3} q}{(2 \pi)^{3}} \vec{q}^{2}\left(\frac{\Lambda_{c}^{2}+m_{\phi}^{*^{2}}}{\Lambda_{c}^{2}+4 E_{K}^{*^{2}}}\right)^{4} \\
& \times \frac{\left(E_{K}^{*}+E_{\bar{K}}^{*}\right)}{E_{K}^{*} E_{\bar{K}}^{*}\left(\left(E_{K}^{*}+E_{\bar{K}}^{*}\right)^{2}-m_{\phi}^{*^{2}}\right)} .
\end{aligned}
$$

To have an estimate for the value of the parameter $\Lambda_{c}$ appearing in the above equation, the form factor is also calculated using the quark pair creation model and the Gaussian wave function for mesons in terms of oscillator constants $[80,96,97]$. Then, the value of the root mean square radii calculated using two different form factors are compared to obtain an expression for $\Lambda_{c}$ in terms of oscillator constants. In Ref. [80] this procedure was used to estimate $\Lambda_{c}$ for the study of the decay width and the in-medium mass of $J / \psi$ mesons. The value of $\Lambda_{c}$ estimated from the expression, $\Lambda_{c}^{2}$ $=32\left(\beta_{D}^{2}+2 \beta_{\psi}^{2}\right)-4 m_{D}^{2}$, was found to be $\approx 2.5 \mathrm{GeV}$. Here, the $D$ meson vacuum mass, $m_{D}$, was taken as $1.867 \mathrm{GeV}$ and the oscillator constants $\beta_{D}$ and $\beta_{\psi}$ were considered as 0.310 and $0.520 \mathrm{GeV}$, respectively. Further, considering the uncertainty in the estimated value, results were shown for different values of $\Lambda_{c}$, i.e., 1,2 , and $3 \mathrm{GeV}$. A similar range is also used in Ref. [19] for the study of $\phi$ mesons. In the present investigation, for the kaon vacuum mass $m_{K}=0.494 \mathrm{GeV}$ and assuming the oscillator constants of $\phi$ and $K$ mesons to be equivalent to those of $J / \psi$ and $D$ mesons, respectively, the estimated value of $\Lambda_{c}$ may increase. Therefore, to understand the dependence of results on the parameter $\Lambda_{c}$, we consider its values in the range 1 to $4 \mathrm{GeV}$.

The value of coupling constant $g_{\phi}$ is determined as 4.539 from the empirical width of the $\phi$ meson in vacuum [98]. The bare mass of the $\phi$ meson is fixed through the constant $g_{\phi}$ and the vacuum mass of the $\phi$ meson, which is taken as $1019.461 \mathrm{MeV}$ [98]. The decay width of the $\phi$ meson is calculated from the imaginary part of the self energy $\operatorname{Im} \Pi_{\phi}^{*}$, and is given in terms of the masses of $\phi, K$, and $\bar{K}$ [20]; so we have

$$
\begin{aligned}
\Gamma_{\phi}^{*}= & \frac{g_{\phi}^{2}}{24 \pi} \frac{1}{m_{\phi}^{*^{*}}}\left[\left(m_{\phi}^{*^{2}}-\left(m_{K}^{*}+m_{\bar{K}}^{*}\right)^{2}\right)\right. \\
& \left.\times\left(m_{\phi}^{*^{2}}-\left(m_{K}^{*}-m_{\bar{K}}^{*}\right)^{2}\right)\right]^{3 / 2} .
\end{aligned}
$$




\section{RESULTS AND DISCUSSIONS}

In this section, we discuss the numerical results obtained in the present work. First, we discuss the in-medium dependence of the scalar fields in Sec. III A. In Sec. III B, density, temperature, isospin asymmetry, and strangeness fraction dependence of $K \bar{K}$ masses are presented, and finally Sec. III C is devoted to presenting the results of $\phi$ meson mass and decay width.

\section{A. The scalar fields of the chiral model in strange hadronic matter}

As discussed in Sec. II A, within the chiral model we have solved the coupled equations of motion of the $\sigma, \zeta, \delta, \chi$, $\omega, \rho$, and $\phi$ mesonic fields. In Fig. 2, we plot the variation of the scalar fields $\sigma$ and $\zeta$, as a function of the baryonic density at finite values of temperature. We also observe the effect of the strangeness fraction and isospin asymmetry in this plot. For every combination of $\eta$ and $f_{s}$, we see that the magnitude of the $\sigma$ and $\zeta$ fields decreases linearly up to nuclear saturation density $\left(\rho_{0}\right)$ and afterward it decreases slowly with further increase in the baryonic density. However, the $\zeta$ field is much less modified as compared to the $\sigma$ field; for example, in the symmetric and nonstrange medium at zero temperature and $\rho_{B}=4 \rho_{0}$, the value of the $\sigma$ field changes by $67 \%$ (as compared with its vacuum value) whereas the $\zeta$ field is modified by $14 \%$ only. Furthermore, considering the effect of the isospin asymmetry of the medium with respect to density, at a particular temperature, the scalar-isoscalar $\sigma$ field shows good $\eta$ dependence whereas the strange scalar-isoscalar $\zeta$ field shows negligible $\eta$ dependence. This is because of the quark content of the respective field: the former mesonic field contains $u$ and $d$ quarks which interact with the medium asymmetry. On the other hand, the latter contains a strange quark pair $(s \bar{s})$. This picture becomes opposite in the strange medium, as, in the presence of hyperons, the strange $\zeta$ field shows appreciable modifications whereas the nonstrange $\sigma$ field shows very little variation. For example, in the symmetric and strange medium at zero temperature and $\rho_{B}=4 \rho_{0}$, the value of the $\zeta$ field changes by $16 \%$ (as compared with its value at $f_{s}=0$ ) whereas the $\sigma$ field is modified by $2 \%$ only. In the symmetric medium, if we move from zero to nonzero temperature, we observe that in the high density regime the value of the $\sigma$ field is modified appreciably in both strange and nonstrange media; at a specific value of density, the magnitude of the $\sigma$ field increases with the increase in temperature. For the nonstrange medium, the strange $\zeta$ field shows negligible $T$ dependence but it turns out to be appreciable in the strange medium. This is because the presence of the strange content in the medium modifies the scalar densities of the hyperons (which depend upon the Fermi distribution functions [10]). As discussed earlier, in the present investigation, the scalar and vector fields are calculated from the coupled equations of motion that contain the expressions of the scalar and vector densities of baryons.

In Fig. 3, the variation of the in-medium $\delta$ and $\chi$ fields are shown for the same medium attributes. As discussed earlier, the asymmetry dependence is introduced in this model by the incorporation of the $\delta$ field and $\eta$ parameter, therefore the $\delta$
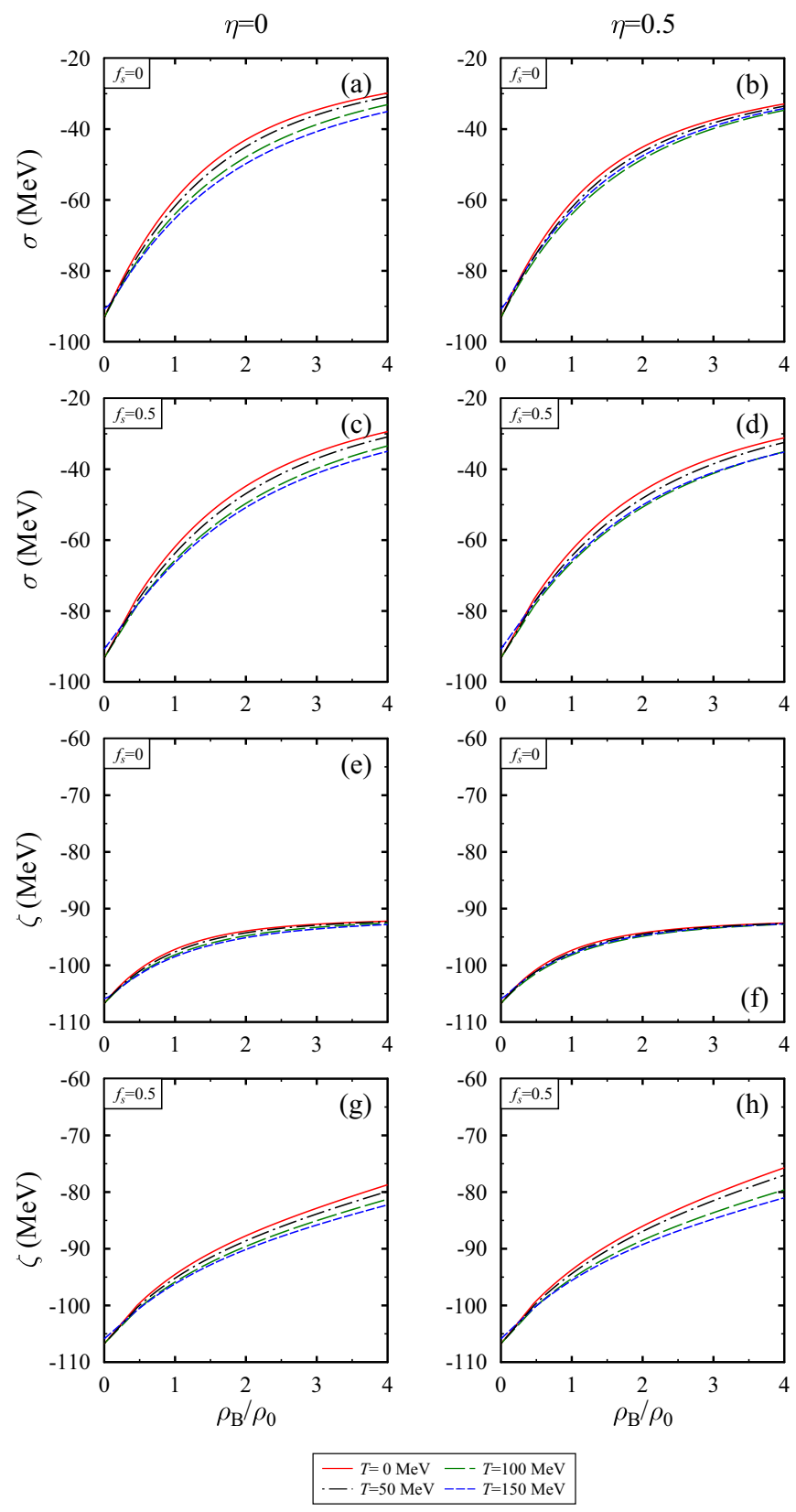

FIG. 2. The in-medium $\sigma$ and $\zeta$ fields in nuclear and hyperonic matter.

field shows appreciable variations in the asymmetric matter but no modifications in the symmetric matter. At $\eta=0.5$ and nonstrange medium, the magnitude of the $\delta$ increases with density, and it is more pronounced in the presence of strange baryons. For finite baryonic density, the $\delta$ field undergoes less drop at high temperature as compared to the $T=0$ situation. The dilaton field $\chi$, which is introduced in the model to mimic the trace anomaly property of QCD [73], varies less with the increase in baryonic density. The magnitude of the $\chi$ field decreases as a function of baryonic density and it shows variations due to asymmetry and strangeness in the high density regime. This is because the $\chi$ field is solved simultaneously in the coupled equations of motion along 

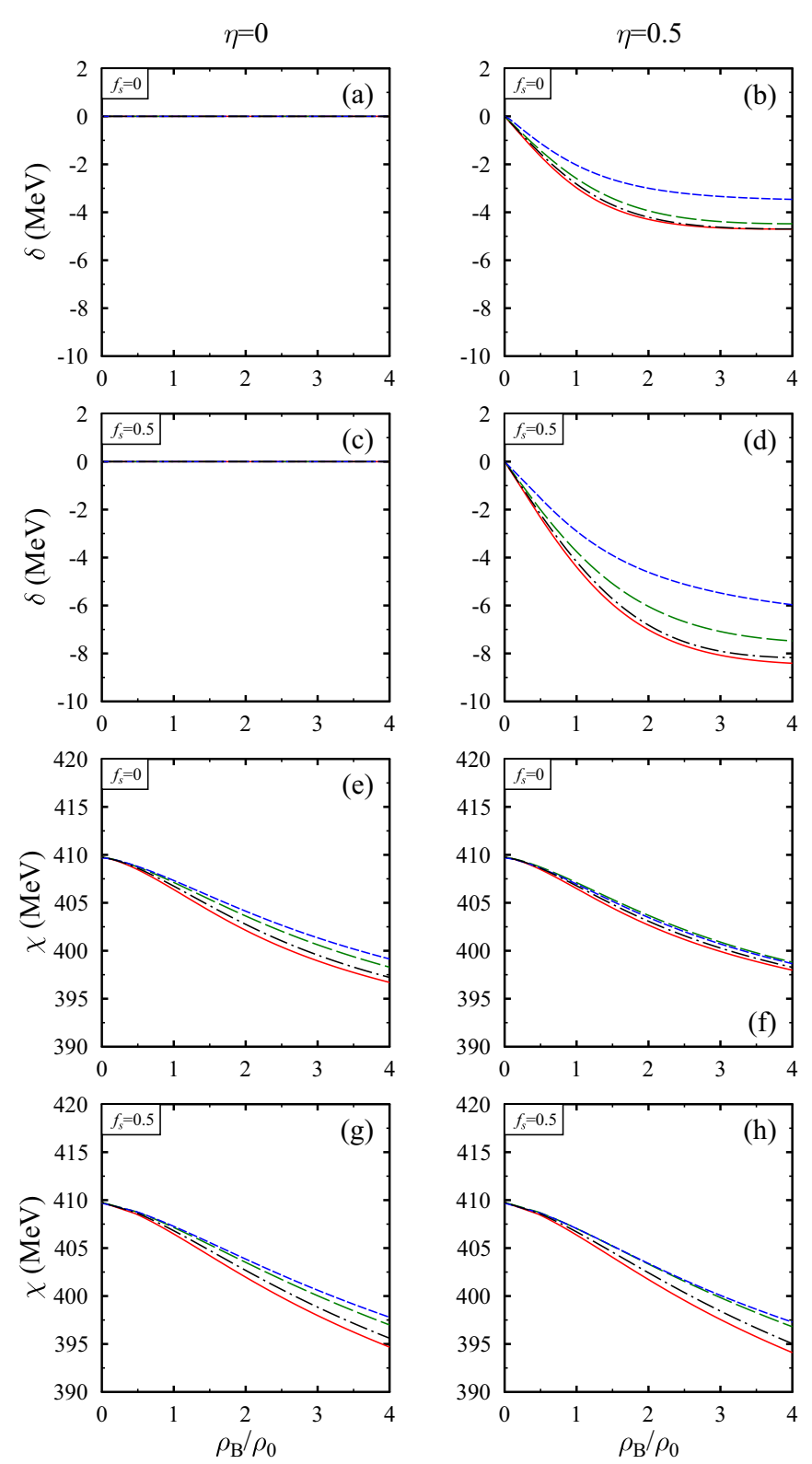

$-T=0 \mathrm{MeV}--T=100 \mathrm{MeV}$ -. $T=50 \mathrm{MeV}---T=150 \mathrm{MeV}$

FIG. 3. The in-medium $\delta$ and $\chi$ fields in nuclear and hyperonic matter. with other medium fields [68]. In the present chiral SU(3) model, the modifications of scalar fields with the medium's temperature are consistent with the results of the chiral quark mean-field model [99].

\section{B. Kaons and antikaons in strange matter at finite temperature}

In this subsection, we discuss the numerical observations of the kaon and antikaon mass in strange hadronic matter. We use the medium induced scalar and vector densities of baryons in the dispersion relation [Eq. (14)] to calculate the in-medium mass of these mesons. In Table II, we list the in-medium masses of the kaons and antikaons for distinct medium parameters. We plot the in-medium masses of $\mathrm{K}^{+}$ and $\bar{K}^{0}$ as a function of baryonic density in Fig. 4. We plot this figure for different values of asymmetry, strangeness, and temperature. In a nonstrange medium, the masses of the $K^{+}$ and $K^{0}$ mesons increase with the increase in the baryonic density. They increases almost linearly for high temperature but comparative slowly for lower temperatures. As discussed earlier, the various terms of Eq. (11) explain the kaon and antikaon baryon interaction. The first term of the Eq. (11) (known as Weinberg-Tomozawa term) gives repulsive contributions to the masses of $K^{+}$and $K^{0}$ mesons [8,59]. The meson exchange term arising from the $\sigma$ and $\delta$ fields is attractive for both $K^{+}$and $K^{0}$ mesons. In the isospin symmetric matter (at a particular value of $f_{s}$ ), the $K^{0}$ mass shows the same behavior as $m_{K^{+}}^{*}$. This is because the $K^{+}$and $K^{0}$ mesons belong to the same isospin doublet. However, in asymmetric nuclear matter, the masses of these mesons do not remain the same because of the asymmetric terms $\left(\rho_{i}-\rho_{j} ; i \neq j\right)$ present in the Weinberg-Tomozawa term and isospin dependent range $\left(d_{2}\right)$ terms. The self-energy of the $K^{+}$meson largely depends upon the scalar and vector densities of the baryons which have positive $\tau_{3}$ values, whereas the self-energy of $K^{0}$ largely depends upon the densities of baryons with negative $\tau_{3}$ values, and due to this the Weinberg-Tomozawa term becomes more repulsive for $K^{0}$ mesons and suppresses the attractive contributions from the $d_{2}$ term. When we move from the nonstrange to the strange medium, the mass of the $K^{+}$meson slightly increases and then decreases with respect to baryonic density for all temperatures. This is because when we go from nonstrange to strange medium, the range term $d_{1}$ becomes more negative whereas the $d_{2}$ term becomes less negative. Therefore the attractive nature of the $d_{1}$ term dominates the

TABLE I. Various parameters used in the present calculations in the strange hadronic matter [9].

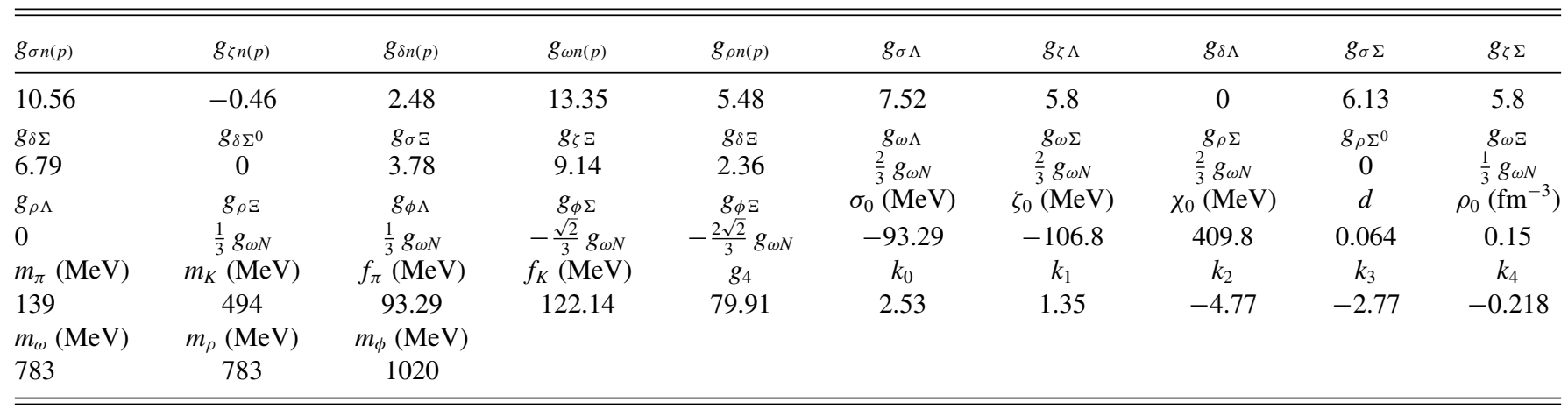


TABLE II. Values of the in-medium masses of $K^{+}, K^{0}, K^{-}$, and $\bar{K}^{0}$ mesons (in units of MeV).

\begin{tabular}{|c|c|c|c|c|c|c|c|c|c|}
\hline & \multirow{2}{*}{$f_{s}$} & \multicolumn{4}{|c|}{$T=50 \mathrm{MeV}$} & \multicolumn{4}{|c|}{$T=150 \mathrm{MeV}$} \\
\hline & & $\rho_{0}$ & $4 \rho_{0}$ & $\rho_{0}$ & $4 \rho_{0}$ & $\rho_{0}$ & $4 \rho_{0}$ & $\rho_{0}$ & $4 \rho_{0}$ \\
\hline \multirow[t]{2}{*}{$m_{K^{+}}^{*}$} & 0 & 524 & 565.1 & 509.5 & 527 & 526.2 & 587.9 & 510.6 & 530.17 \\
\hline & 0.5 & 503.9 & 470.3 & 506.6 & 498.2 & 506 & 491.7 & 498.9 & 475.2 \\
\hline$m_{K^{0}}^{*}$ & 0 & 524 & 565.1 & 532.7 & 613.4 & 526.2 & 587.9 & 534.5 & 625.1 \\
\hline \multirow[t]{2}{*}{$m_{K^{-}}^{*}$} & 0 & 458.7 & 314.9 & 466.7 & 352.2 & 461.8 & 326.2 & 468.5 & 355.1 \\
\hline & 0.5 & 473.2 & 344.1 & 453.6 & 302.1 & 475.6 & 360.3 & 470 & 353 \\
\hline \multirow[t]{2}{*}{$m_{\bar{K}^{0}}^{*}$} & 0 & 458.7 & 314.9 & 444.5 & 283.3 & 461.8 & 326.2 & 446.6 & 287.7 \\
\hline & 0.5 & 473.2 & 344.1 & 487.5 & 366.8 & 475.6 & 360.3 & 474.3 & 347.96 \\
\hline
\end{tabular}

range terms and, overall, due to this the masses of $K$ and $K^{0}$ decrease in the strange medium. The temperature effects on the masses of $K^{+}$and $K^{0}$ mesons become smaller for non-strange asymmetric nuclear matter and for the strange asymmetric matter, it shows appreciable modifications. In the regime of high density, the masses of $K$ mesons decrease with the decrease in temperature. The above behavior can be explained on the basis of the temperature dependence of baryon scalar densities. As we increase the medium's temperature, the attractive contributions from the range terms start decreasing.

Similarly, we plot the in-medium mass of antikaons $K^{-}$ and $\bar{K}^{0}$ in Fig. 5. For the nonstrange medium, we observe an opposite behavior in the masses of antikaons as compared to kaons because of the attractive contributions from the Weinberg-Tomozawa term. Furthermore, since $K^{-}$and $\bar{K}^{0}$ mesons also belong to the isospin doublet, the masses of these mesons do not show any difference in the symmetric nuclear matter (for a particular value of strangeness). The masses of $K^{-}$and $\bar{K}^{0}$ mesons show less impact of temperature in the nonstrange asymmetric baryonic matter but for strange asymmetric medium it shows an appreciable variation with temperature. The explanation lies in the same fact as was discussed for $K$ mesons in the previous paragraph. Using the quark meson coupling model, in Ref. [19], the mass of $K$ meson is studied in the nonstrange symmetric nuclear matter at zero temperature. In this article, the authors observed that the mass of kaons decreases as a function of baryonic density. Moreover, in Ref. [20], using relativistic transport model Li et al. studied the mass of the $K(\bar{K})$ meson along with the masses of the $\rho$ and $\phi$ mesons in the hadronic medium which contains nucleons, pions, and deltas. They observed that the mass of kaons increases with the increase in baryonic density while the antikaons' mass decreases. Using the kaon and antikaon in-medium mass, one can also calculate the in-medium optical potential for finite momentum situations via the relation $U_{K(\bar{K})}^{*}(\omega, k)=\omega(k)-m_{K(\bar{K})}[8,59]$.

At very low densities, where the impulse approximation is valid $\left(U \approx-4 \pi a_{\bar{K} N} \rho\right)$, the optical potential for antikaons will come out to be positive because of the repulsive value of scattering length at the $\bar{K} N$ threshold [100]. However, with the approach of mean-field calculations, attractive potentials will obtain at very low densities [19,59,93-95]. This is because the generation of $\Lambda(1405)$ is not considered in these calculations, though it is usually considered in the coupled channel approach [46,49,55-57]. However, the work on in-medium properties of kaons and antikaons using the mean-field approach is an alternative and has been used by various groups [19,59,93-95], especially for the study of optical potentials at high baryonic densities which may exist in the core of neutron stars and may also be produced in the CBM experiment of the FAIR project. The kaonic atom data also demand the attractive potential for antikaons in nuclear medium. The attractive potentials for antikaons in the coupled channel approach are obtained even at low densities (which is required to explain kaonic atom data) due to inclusion of the medium effect through Pauli blocking and self-consistent consideration of the $\bar{K}$ self-energy [46,49,55-58].

\section{The in-medium mass and decay width of the $\phi$ meson}

The $\phi$ meson mass is calculated through the in-medium self-energy of the $\phi$ meson at the one-loop level (see Sec. II C). In the previous works of Refs. [19,35], the loop integral was solved under the assumption $m_{K}^{*}=m_{\bar{K}}^{*}$, but in the present work the self-energy of the $\phi$ meson is calculated by solving the regularized loop integral in the presence of the medium modified kaons and antikaons where they behave differently, as discussed in Sec. III B. Note that the temperature dependence of the $\phi$ meson mass and decay width in our present calculations is evaluated through the temperature dependence of the kaon and antikaon masses. In Fig. 6, we show the in-medium mass of the $\phi$ meson as a function of baryonic density by considering the effect of isospin asymmetry, strangeness, and temperature for cutoff parameter $\Lambda_{c}$ $=3 \mathrm{GeV}$. The medium induced masses for the different parameter combinations are tabulated in Table III, From Fig. 6, we observe that the mass of the $\phi$ meson decreases as a function of the medium density. For all permutations of $\eta$ and $f_{s}$, we see that if the temperature of the medium is decreased then the mass of the $\phi$ meson also decreases, which reflects the temperature dependence of kaon and antikaon masses. Furthermore, the effect of temperature is more visible in the symmetric matter than in the asymmetric matter. Moreover, the increase in strange quarks in the hadronic medium leads to more decrease in $m_{\phi}^{*}$. The in-medium behavior of the $\phi$ meson mass reflects the in-medium masses of $K$ and $\bar{K}$ as 

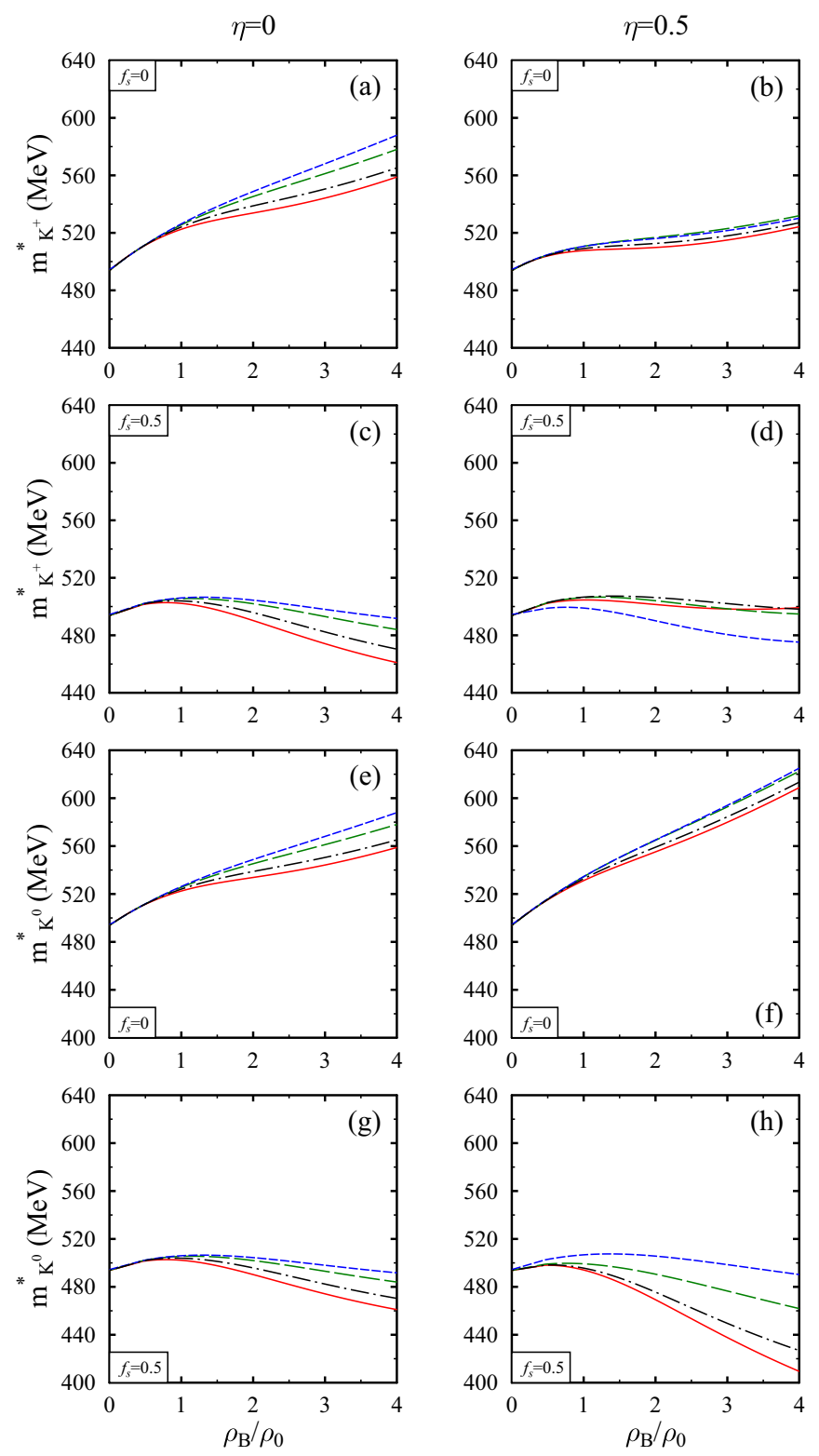

\begin{tabular}{|l|}
$-T=0 \mathrm{MeV}--T=100 \mathrm{MeV}$ \\
$-\longrightarrow T=50 \mathrm{MeV}--T=150 \mathrm{MeV}$
\end{tabular}

FIG. 4. The in-medium mass of isospin doublet $\left(K^{+}, K^{0}\right)$ in nuclear and hyperonic matter.

the self-energy of the $\phi$ meson loop is calculated using these medium modified entities. To understand the dependence of the in-medium mass of the $\phi$ meson on the cutoff parameter $\Lambda_{c}$, in Fig. 7 the results are shown for $\Lambda_{c}=1,2,3$. and 4 $\mathrm{GeV}$. We observed that in the nonstrange and strange matter the trend of the in-medium mass $m_{\phi}^{\star}$ remains the same with respect to the baryonic density but it decreases more as we increase the value of the parameter $\Lambda_{c}$ from 1 to $4 \mathrm{GeV}$. This is consistent with the observations of Ref. [19] where, using the in-medium $\phi$ meson self-energy in the nonstrange symmetric nuclear matter, Cobos-Martinez et al. studied the medium induced mass of the $\phi$ meson. The authors observed that the mass of the $\phi$ meson decreases with the increase of


\begin{tabular}{|l|}
\hline$-T=0 \mathrm{MeV}--T=100 \mathrm{MeV}$ \\
- - $T=50 \mathrm{MeV}---T=150 \mathrm{MeV}$
\end{tabular}

FIG. 5. The in-medium mass of isospin doublet $\left(K^{-}, \bar{K}^{0}\right)$ in nuclear and hyperonic matter.

the nucleonic density and they plotted the results for different values of the cutoff parameter. At $\Lambda_{c}=3 \mathrm{GeV}$ and $\rho_{B}=\rho_{0}$, they observed a $25 \mathrm{MeV}$ decrement in the $\phi$ meson mass whereas we observed a $2.59 \mathrm{MeV}$ drop. This is because in our calculations kaons and antikaons behave differently in the medium, for example, as we discussed earlier, the WeinbergTomozawa term gives the repulsive contribution to $K$ meson mass and the attractive contribution to $\bar{K}$ meson mass. The values of $m_{K}^{*}$ and $m_{\bar{K}}^{*}$ are observed as 522.49 and $456.75 \mathrm{MeV}$ in symmetric nuclear matter at density $\rho_{0}$ and temperature $T=0$.

However, in Ref. [19], the difference in in-medium masses of kaons and antikaons is not considered in the calculations, and at $\rho_{0}$ the mass $m_{K}^{*}$ is simply considered as $430 \mathrm{MeV}$. In 

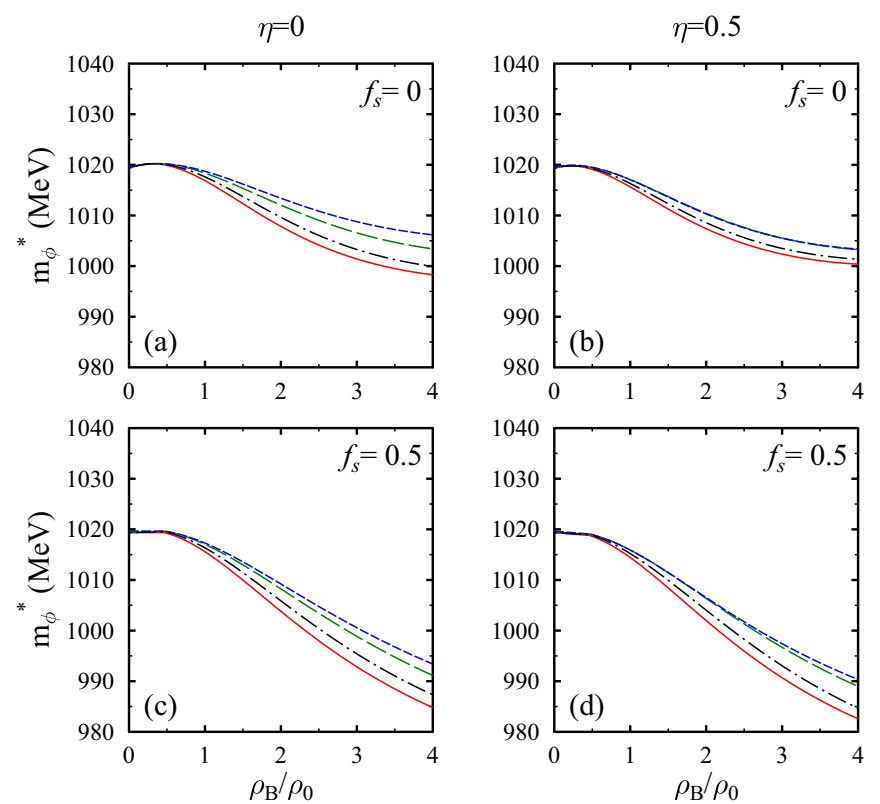

\begin{tabular}{|l|}
\hline$T=0 \mathrm{MeV}--T=100 \mathrm{MeV}$ \\
$-T=50 \mathrm{MeV}--T=150 \mathrm{MeV}$
\end{tabular}

FIG. 6. The in-medium mass of the $\phi$ meson in nuclear and hyperonic matter for the cutoff parameter $\Lambda_{c}=3 \mathrm{GeV}$.

our article, we observed less downward shift in the $K$ and $\bar{K}$ masses and therefore a little drop in the $\phi$ mass. Using QCD sum rules at zero temperature, Klingl et al. calculated $1 \%$ drop in $\phi$ mass at nuclear saturation density [60]. Furthermore, using the unification of the chiral SU(3) model and QCD sum rules, the author studied the in-medium mass of the $\phi$ meson in the asymmetric strange matter at zero temperature [13] and observed a very small drop. The author reported a mass shift of about $20 \mathrm{MeV}$ at a density of $5 \rho_{0}$ in the nuclear medium.

In Fig. 8, we plot the in-medium partial decay width of the $\phi$ meson decaying into $K \bar{K}$ pairs for $\Lambda_{c}=3 \mathrm{GeV}$. The formula for the decay width is derived by extracting the imaginary part of the loop integral. The medium modified value of $\Gamma_{\phi}^{*}$ is also listed in the Table III along with $m_{\phi}^{*}$. In this figure, we observe that the partial decay width increases (broadens) with the increase in the baryonic density for all cases of strangeness, temperature, and isospin asymmetry. The decay width reflects opposite behavior, as was observed for $m_{\phi}^{*}$. However, in the asymmetric matter, we observe the
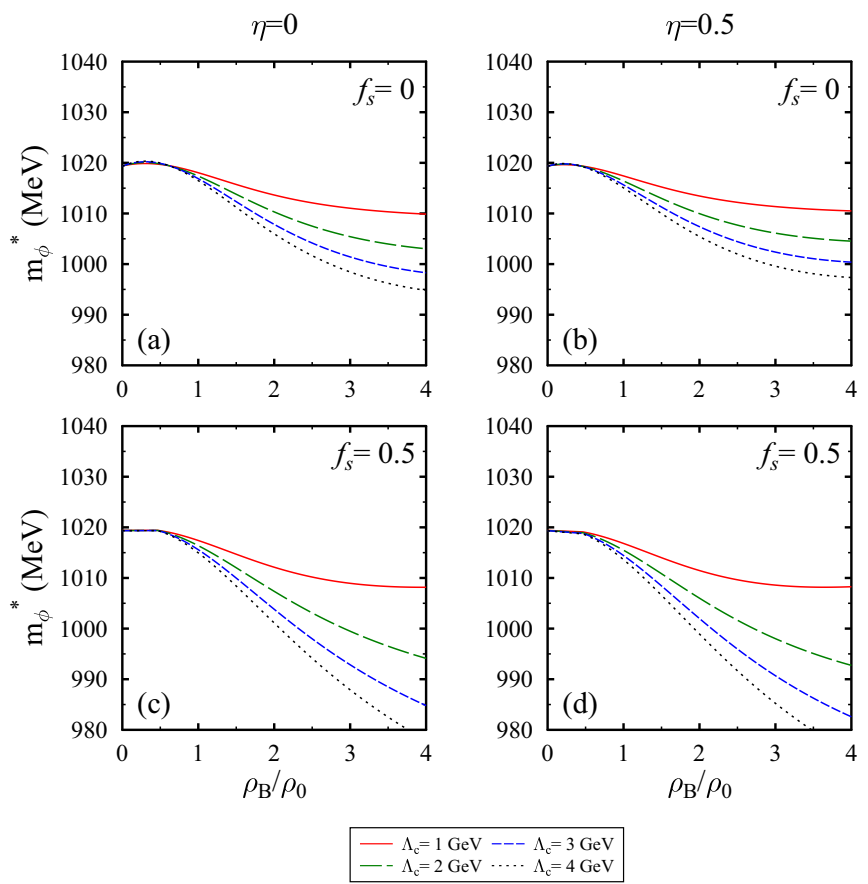

FIG. 7. The in-medium mass of the $\phi$ meson in nuclear and hyperonic matter for different values of the parameter $\Lambda_{c}$ at $T=0$ $\mathrm{MeV}$.

temperature effects to be less appreciable, which was also the case for $m_{\phi}^{*}$. But here, in the decay width case, at a particular value of the baryonic density the in-medium decay width increases more for low temperature whereas it was opposite for the mass of the $\phi$ meson. The impact of strange matter on the $\phi$ mesons leads to more increase in the decay width with baryonic density. This is because of the peculiar behavior of $K$ and $\bar{K}$ mesons in strange matter. This highlights the importance of the study of strangeness effects on properties of the $\phi$ mesons.

The dependence of the decay width of the $\phi$ meson on the cutoff parameter $\Lambda_{c}$ is shown in Fig. 9 and it is observed that the values of decay width decrease as $\Lambda_{c}$ is changed from 1 to $4 \mathrm{GeV}$, which is again consistent with the results of Ref. [19]. Moreover, Cabrera et al. described the additional $\phi$ meson decay width, which does not accommodate the effective masses of kaons and antikaons [28-30]. The additional $\bar{K}$ coupling due to $\Lambda$ resonance impacts the decay of the

TABLE III. Values of the medium induced $\phi$-meson mass and partial decay width (MeV) for the $\phi \rightarrow K \bar{K}$ process for different parameters of the medium. The values are tabulated for $\Lambda_{c}=3 \mathrm{GeV}$.

\begin{tabular}{|c|c|c|c|c|c|c|c|c|c|}
\hline & $f_{s}$ & \multicolumn{2}{|c|}{$\eta=0$} & \multicolumn{2}{|c|}{$\eta=0.5$} & \multicolumn{2}{|c|}{$\eta=0$} & \multicolumn{2}{|c|}{$\eta=0.5$} \\
\hline \multirow[t]{2}{*}{$m_{\phi}^{*}$} & 0 & 1017.6 & 999.9 & 1016.3 & 1001.3 & 1018.7 & 1006.1 & 1017 & 1003.3 \\
\hline & 0.5 & 1016.3 & 987 & 1015 & 984 & 1017 & 993 & 1016 & 990 \\
\hline$\Gamma_{\phi}^{*}$ & 0 & 4.8 & 26.5 & 5.8 & 24.4 & 3.9 & 18 & 5.2 & 21.6 \\
\hline
\end{tabular}





\begin{tabular}{|l|}
\hline$T=0 \mathrm{MeV}--T=100 \mathrm{MeV}$ \\
$-T=50 \mathrm{MeV}---T=150 \mathrm{MeV}$ \\
\hline
\end{tabular}

FIG. 8. The in-medium decay width of the $\phi \rightarrow K \bar{K}$ channel in nuclear and hyperonic matter for the cutoff parameter $\Lambda_{c}=3 \mathrm{GeV}$.

$\phi \rightarrow K \bar{K}$ process, followed by $\bar{K} \rightarrow \Lambda(\Sigma) h$, or the decay $\phi N \rightarrow K \Lambda(\Sigma)$. The $P$-wave $\bar{K}$ self-energy originates from the kaon coupling to the $\Lambda h, \Sigma h$, and $\Sigma^{*} h$ hyperons [28]. Therefore, to get a clearer picture, one has to take into account the effect of an additional $\bar{K}$ coupling which modifies the selfenergy and hence the optical potential of $\bar{K}$. We will study this effect in the near future by including the additional kaon selfenergy in our model. As per other theoretical and experimental investigations discussed in the Introduction, the decrement (increment) in the $\phi$ meson mass (decay width) in the present investigation is consistent with the observations of existing literature with some distinctions. The cause for these distinctions may lie in the estimation of the kaon-antikaon loop contributions from different approaches. On the application side, by utilizing the decay width, the production of $\phi$ mesons in $p N$ collisions can be measured [101]. The comparison of the experimental data with model calculations will clarify the absorption, production, and momentum dependence of the $\phi$ meson in the hadronic medium [102,103]. In [103] the momentum dependence of the nuclear transparency ratio and the in-medium decay width of $\phi$ mesons was investigated. The extracted values of the decay width are found to have significant momentum dependence.

\section{SUMMARY}

To summarize, using an effective Lagrangian approach we calculated the medium modified mass and decay width of the $\phi$ meson by employing the in-medium $K$ and $\bar{K}$ masses from the chiral SU(3) model. We have calculated these properties up to four times the nuclear saturation density and in
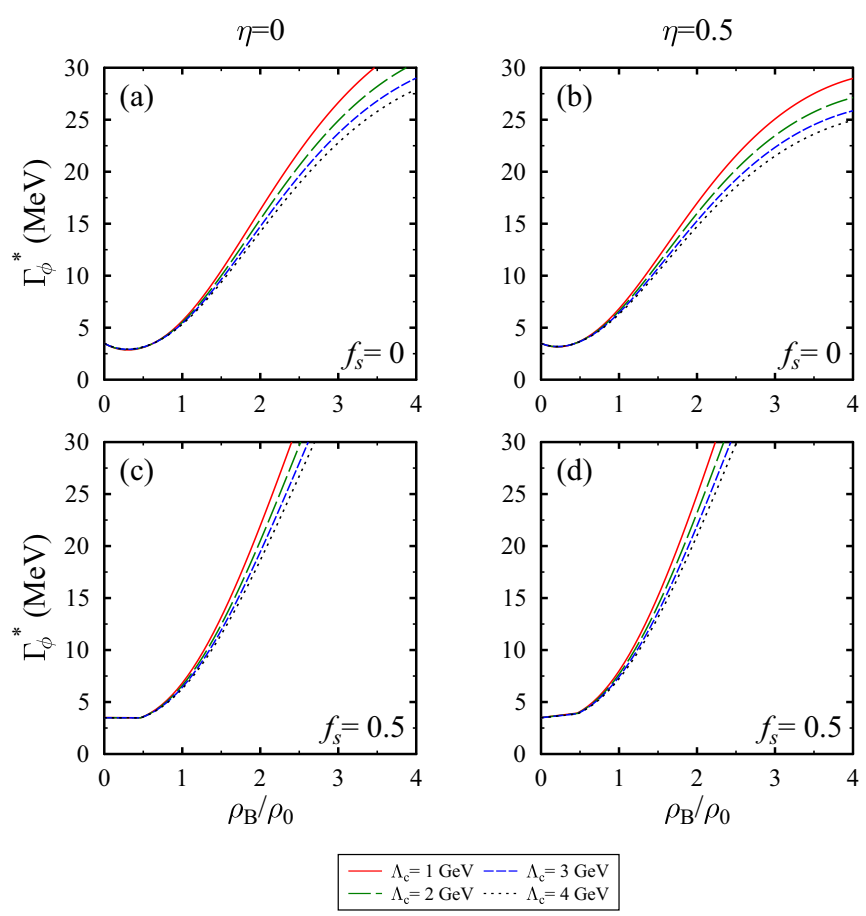

FIG. 9. The in-medium decay width of the $\phi \rightarrow K \bar{K}$ channel the nuclear and hyperonic matter for different values of the parameter $\Lambda_{c}$ at $T=0 \mathrm{MeV}$.

the medium; we considered the nucleons and hyperons as degrees of freedom. At finite temperature, we observed the appreciable effect of the strangeness on the in-medium $K$ and $\bar{K}$ mesons. The kaon baryon interactions in the strange medium lead to a decrease in the $K$ and $\bar{K}$ masses. The mass of antikaons decreases more appreciably than that of kaons in the medium. Despite a significant drop in the $K$ and $\bar{K}$ masses, we observed a small downward mass shift in the in-medium mass of the $\phi$ meson. The impact of temperature becomes smaller in the asymmetric baryonic medium whereas it becomes high in the symmetric medium. On the other hand, in the same medium, the decay width shows broadening and decreases with the increase in the strange content of the medium. In the extension of our work, we will study the appreciable effects on $\phi$ mesons such as strangeness enhancement in the $\phi$ mesic nuclei [104] and absorption and production of the $\phi$ meson in the hadronic medium [101,102]. It requires future experimental efforts to understand the medium induced changes in the $\phi$ meson properties in the strange medium. A more systematic study, by the J-PARC E16 Collaboration [105], is intended to explore the mass shift of vector mesons with higher statistics. There is also a proposal at J-Lab (following the $12 \mathrm{GeV}$ upgrade) to study the binding of helium nuclei with $\phi$ and $\eta$ mesons [36]. Furthermore, the $K^{+} / K^{0}$ and $K^{-} / \bar{K}^{0}$ ratios for different isospins of the beam and target are promising observables to study the asymmetry effects in the CBM experiment at the future project FAIR at GSI, Germany, and at the Rare Isotope Accelerator (RIA) laboratory in the USA [8]. 


\section{ACKNOWLEDGMENTS}

One of the authors (R.K.) sincerely acknowledges the support towards this work from the Ministry of Science and
Human Resources Development (MHRD), Government of India via an Institute fellowship under the National Institute of Technology, Jalandhar.
[1] L. Tolos and L. Fabbietti, Prog. Part. Nucl. Phys. 112, 103770 (2020).

[2] Holzenkamp et al., Nucl. Phys. A 500, 485 (1989).

[3] S. Petschauer et al., Eur. Phys. J. A 52, 15 (2016).

[4] Haidenbauer et al., Eur. Phys. J. A 55, 23 (2019).

[5] R. Chhabra and A. Kumar, Eur. Phys. J. A 53, 105 (2017).

[6] R. Chhabra and A. Kumar, Phys. Rev. C 98, 025205 (2018).

[7] A. Kumar and R. Chhabra, Phys. Rev. C 92, 035208 (2015).

[8] A. Mishra et al., Eur. Phys, J. A 41, 205 (2009).

[9] A. Kumar and A. Mishra, Eur. Phys. J. A 47, 164 (2011).

[10] R. Kumar and A. Kumar, Eur. Phys. J. C 79, 403 (2019).

[11] R. Rapp et al., Prog. Part. Nucl. Phys. 65, 209 (2010).

[12] H. J. Kim and P. Gubler, Phys. Lett. B 805, 135412 (2020).

[13] A. Mishra, Phys. Rev. C 91, 035201 (2015).

[14] A. Mishra, A. Kumar, P. Parui, and S. De, Phys. Rev. C 100, 015207 (2019).

[15] Shivam and A. Kumar, Eur. Phys. J. Plus 134, 592 (2019).

[16] S. Leupold et al., Int. J. Mod. Phys. E 19, 147 (2010).

[17] R. S. Hayano and T. Hatsuda, Rev. Mod. Phys. 82, 2949 (2010).

[18] G. Krein, in XIth Conference on Quark Confinement and Hadron Spectrum, 8-12 September 2014, Saint Petersburg, edited by A. Andrianov, N. Brambilla, V. Kim, and S. Kolevatov, AIP Conf. Proc. No. 1701 (AIP, New York, 2016), p. 020012.

[19] J. J. Cobos-Martinez et al., Phys. Lett. B 771, 113 (2017).

[20] G. Q. Li and C. M. Ko, Nucl. Phys. A 582, 731 (1995).

[21] C. M. Ko, P. Lévai, X. J. Qiu, and C.T. Li, Phys. Rev. C 45, 1400 (1992).

[22] C. Gale and J. Kapusta, Phys. Rev. C 35, 2107 (1987).

[23] L. H. Xia et al., Nucl. Phys. A 485, 721 (1988).

[24] C. M. Ko et al., Phys. Lett. B 231, 16 (1989).

[25] C. L. Korpa et al., Phys. Lett. B 246, 333 (1990).

[26] L. Xiong, J. Q. Wu, Z. G. Wu, C. M. Ko, and J. H. Shi, Phys. Rev. C 41, R1355(R) (1990).

[27] L. Xiong et al., Nucl. Phys. A 512, 772 (1990).

[28] D. Cabrera et al., Phys. Rev. C 67, 045203 (2003).

[29] D. Cabrera et al., Phys. Rev. C 96, 034618 (2017).

[30] D. Cabrera et al., Phys. Rev. C 95, 015201 (2017).

[31] A. Sibirtsev et al., Eur. Phys. J. A 29, 209 (2006).

[32] P. Gubler and K. Ohtani, Phys. Rev. D 90, 094002 (2014).

[33] A. Bottino et al., Astropart. Phys. 18, 205 (2002).

[34] J. Ellis, K.A. Olive, and C. Savage, Phys. Rev. D 77, 065026 (2008).

[35] J. J. Cobos-Martinez et al., Phys. Rev. C 96, 035201 (2017).

[36] https://www.jlab.org/exp_prog/PACpage/PAC42/PAC42_ FINAL_Report.pdf.

[37] P. Buhler et al., Prog. Theor. Phys. Suppl. 186, 337 (2010).

[38] H. Ohnishi et al., Acta Phys. Pol. B 45, 819 (2014).

[39] D. B. Kaplan and A. E. Nelson, Phys. Lett. B 175, 57 (1986); A. E. Nelson and D. B. Kaplan, ibid. 192, 193 (1987).

[40] G. Q. Li et al., Nucl. Phys. A 625, 372 (1997).

[41] C. M. Ko, J. Phys. G 27, 327 (2001).

[42] S. Pal, C. M. Ko, and Z. -w. Lin, Phys. Rev. C 64, 042201(R) (2001).
[43] W. Cassing et al., Nucl. Phys. A 614, 415 (1997).

[44] E. L. Bratkovskaya et al., Nucl. Phys. A 622, 593 (1997).

[45] W. Cassing and E. L. Bratkovskaya, Phys. Rep. 308, 65 (1999).

[46] M. Lutz, Phys. Lett. B 426, 12 (1998).

[47] M. Lutz and E. E. Kolomeitsev, Nucl. Phys. A 700, 193 (2002).

[48] M. Lutz and C. L. Korpa, Nucl. Phys. A 700, 309 (2002).

[49] A. Ramos and E. Oset, Nucl. Phys. A 671, 481 (2000).

[50] L. Tolós, A. Ramos, and A. Polls, Phys. Rev. C 65, 054907 (2002).

[51] F. Laue et al. (KaoS Collaboration), Phys. Rev. Lett. 82, 1640 (1999).

[52] M. Menzel et al. (KaoS Collaboration), Phys. Lett. B 495, 26 (2000).

[53] C. Sturm et al. (KaoS Collaboration), Phys. Rev. Lett. 86, 39 (2001).

[54] A. Förster et al. (KaoS Collaboration), J. Phys. G 28, 2011 (2002).

[55] L. Tolos et al., Nucl. Phys. A 690, 547 (2001).

[56] L. Tolós, A. Ramos, and E. Oset, Phys. Rev. C 74, 015203 (2006).

[57] M. F. M. Lutz et al., Nucl. Phys. A 808, 124 (2008).

[58] L. Tolos, D. Cabrera, and A. Ramos, Phys. Rev. C 78, 045205 (2008).

[59] A. Mishra, S. Schramm, and W. Greiner, Phys. Rev. C 78, 024901 (2008).

[60] F. Klingl, T. Waas, and W. Weise, Phys. Lett. B 431, 254 (1998).

[61] T. Hatsuda and S. H. Lee, Phys. Rev. C 46, R34(R) (1992).

[62] T. Hatsuda, H. Shiomi, and H. Kuwabara, Prog. Theor. Phys. 95, 1009 (1996).

[63] E. Oset and A. Ramos, Nucl. Phys. A 679, 616 (2001).

[64] R. Muto et al. (KEK-PS-E325 Collaboration), Phys. Rev. Lett. 98, 042501 (2007)

[65] T. Ishikawa et al., Phys. Lett. B 608, 215 (2005).

[66] T. Mibe et al. (CLAS Collaboration), Phys. Rev. C 76, 052202 (2007).

[67] X. Qian et al. (CLAS Collaboration), Phys. Lett. B 680, 417 (2009).

[68] R. Kumar and A. Kumar, Phys. Rev. C 101, 015202 (2020).

[69] P. Papazoglou, D. Zschiesche, S. Schramm, J. SchaffnerBielich, H. Stöcker, and W. Greiner, Phys. Rev. C 59, 411 (1999).

[70] S. Reddy P., A. Jahan C. S., N. Dhale, A. Mishra, J. SchaffnerBielich, Phys. Rev. C 97, 065208 (2018).

[71] R. N. Mishra, H. S. Sahoo, P. K. Panda, N. Barik, and T. Frederico, Phys. Rev. C 98, 019903 (2018).

[72] A. Kumar, Adv. High Energy Phys. 2014, 549726 (2014).

[73] A. Kumar and A. Mishra, Phys. Rev. C 82, 045207 (2010).

[74] R. Kumar and A. Kumar, Chin. Phys. C 43, 124109 (2019).

[75] A. Mishra et. al., Eur. Phys. J. A 55, 107 (2019).

[76] A. Mishra, K. Balazs, D. Zschiesche, S. Schramm, H. Stocker, and W. Greiner, Phys. Rev. C 69, 024903 (2004).

[77] A. Mishra, E. L. Bratkovskaya, J. Schaffner-Bielich, S. Schramm, and H. Stöcker, Phys. Rev. C 70, 044904 (2004). 
[78] A. Mishra and S. Schramm, Phys. Rev. C 74, 064904 (2006).

[79] R. Kumar et al., arXiv:2004.01379.

[80] G. Krein et al., Phys. Lett. B 697, 136 (2011).

[81] S. Weinberg, Phys. Rev. 166, 1568 (1968).

[82] S. Coleman et al., Phys. Rev. 177, 2239 (1969).

[83] W. A. Bardeen and B. W. Lee, Phys. Rev. 177, 2389 (1969).

[84] D. Zschiesche, Description of hot, dense, and strange hadronic matter in a chiral $S U(3)_{L} \times S U(3)_{R} \sigma$-model, Diploma thesis, Goethe University Frankfurt (1997).

[85] I. Zakout, H. R. Jaqaman, S. Pal, H. Stöcker, and W. Greiner, Phys. Rev. C 61, 055208 (2000).

[86] S. Kubis and M. Kutschera, Phys. Lett. B 399, 191 (1997).

[87] F. Hofmann, C. M. Keil, and H. Lenske, Phys. Rev. C 64, 034314 (2001).

[88] P. Wang et al., Nucl. Phys. A 688, 791 (2001).

[89] J. J. Sakurai, Currents and Mesons (University of Chicago Press, Chicago, 1969).

[90] G. Mao, P. Papazoglou, S. Hofmann, S. Schramm, H. Stöcker, and W. Greiner, Phys. Rev. C 59, 3381 (1999).

[91] T. Barnes and E. S. Swanson, Phys. Rev. C 49, 1166 (1994).

[92] F. Klingl et al., Z. Phys. A 356, 193 (1996).
[93] D. P. Menezes, P. K. Panda, and C. Providencia, Phys. Rev. C 72, 035802 (2005).

[94] S. Banik and D. Bandyopadhyay, Phys. Rev. C 63, 035802 (2001).

[95] N. K. Glendenning and J. Schaffner-Bielich, Phys. Rev. Lett. 81, 4564 (1998).

[96] F. E. Close and E. S. Swanson, Phys. Rev. D 72, 094004 (2005).

[97] S. Godfrey and N. Isgur, Phys. Rev. D 32, 189 (1985).

[98] The Review of Particle Physics, 2014-2015, K. A. Olive et al. (Particle Data Group), Chin. Phys. C, 38, 090001 (2014) and 2015 update, http://pdg.lbl.gov/.

[99] P. Wang et al., Commun. Theor. Phys. 36, 71 (2001).

[100] V. Koch, Phys. Lett. B 337, 7 (1994).

[101] A. Polyanskiy et al., Phys. Lett. B 695, 74 (2011).

[102] E. Ya. Paryev, Chin. Phys. C 42, 084101 (2018).

[103] M. Hartmann et al., Phys. Rev. C 85, 035206 (2012).

[104] http://j-parc.jp/researcher/Hadron/en/pac_0907/pdf/Ohnishi. pdf.

[105] http://rarfaxp.riken.go.jp/ yokkaich/paper/jparc-proposal0604.pdf. 